\title{
DETERMINANTES DEL RENDIMIENTO ACADÉMICO EN NEIVA: UNA APROXIMACIÓN A TRAVÉS DE UN MODELO MULTINIVEL
}

\author{
Oscar Hernán Cerquera losada * \\ José Jardani Giraldo Uribe ${ }^{\dagger}$ \\ Guillermo Leon Cordoba Nieto $\ddagger$
}

\begin{abstract}
Resumen
Este trabajo analiza los determinantes del rendimiento académico de la educación media para las áreas de lenguaje y matemáticas en Neiva, Colombia, durante el periodo 2008-2011. Se desarrollaron métodos de investigación etnográficos, y también se estimó un modelo multinivel con el que se busca determinar el peso relativo de la escuela y las características individuales en el rendimiento académico. Se encontró que el efecto colegio tiene mayor peso sobre el rendimiento académico que el efecto estudiante.
\end{abstract}

Palavras-chave: calidad educativa, rendimiento académico, prueba Saber $11^{\circ}$, modelos multinivel, factor escuela, factor estudiante.

\begin{abstract}
This paper analyzes the determinants of the academic performance of secondary education for the areas of language and mathematics in Neiva, Colombia, during the period 2008-2011. Ethnographic research methods were developed, and a multilevel model was also estimated with which it is sought to determine the relative weight of the school and the individual characteristics in the academic performance. It was found that the college effect has more weight on the academic performance than the student effect.
\end{abstract}

Keywords: educational quality, academic performance, Saber $11^{\circ}$ test, multilevel models, school factor, student factor.

JEL classification: C39

DOI: http : / dx.doi .org/10.11606/1980-5330/ea112987

\footnotetext{
${ }^{*}$ Magister en Economía por la Universidad de Buenos Aires, Economista de la Universidad Surcolombiana. Docente de planta del programa de Economía de la Universidad Surcolombiana. Email: oscar.cerquera@usco.edu.co.

† Doctorado en Desarrollo Sostenible, Universidad de Manizales. Magister en Administración Económica y Financiera. Docente de planta del programa de Administración de Empresas de la Universidad Surcolombiana. email: jose jardani@usco.edu.co.

‡ Doctorado en Economía, Universidad de la Amistad de Los Pueblos Patricio Lumumba, Moscu. Economista de la misma Universidad. Docente de planta del programa de Economía de la Universidad Surcolombiana. Email: guillermoleon@usco.edu.co.
} 


\section{Introducción}

El objetivo de cualquier sociedad del mundo es buscar un crecimiento económico sostenible en el largo plazo, interesados siempre por el mejoramiento del bienestar de toda la población. Algunos autores enfatizan que el crecimiento se genera en el seno de la economía bien sea por el capital humano (Lucas Jr 1988), por la existencia de rendimientos constantes a escala en la función de producción (Rebelo 1991), por las externalidades tecnológicas (Romer 1986), o por la ausencia de distorsiones en el mercado (Easterly 1993). Lucas, propone que el factor fundamental para lograr un aumento continuo del producto en el largo plazo, es la cualificación del capital humano, mayores años de estudio se relacionan con un mayor nivel de producción per cápita (productividad).

En general la educación es analizada desde tres perspectivas: i) Cobertura, ii) Eficiencia y iii) Calidad. Este artículo se ubica en el último punto, pero no abarca su concepto en general, sino que se limita al tema del rendimiento académico. Por lo anterior, en este artículo se pretende determinar los factores que influyen en el rendimiento académico de la educación media en Neiva, la cual es una ciudad intermedia de Colombia caracterizada por presentar un bajo desarrollo económico y social. El análisis se realizó teniendo en cuenta los resultados de la prueba Saber $11^{\circ}$ para las áreas de matemáticas y lenguaje de los estudiantes de las instituciones de educación pública de la ciudad.

Para resolver el problema propuesto se utilizó dos métodos. Por un lado, se desarrolló el enfoque teórico de la Función de Producción Educativa propuesto por Hanushek (1986), en donde el producto final está representado por el puntaje obtenido por el estudiante en las Pruebas Saber $11^{\circ}$ para las áreas de lenguaje y matemáticas. Los insumos o factores productivos, están representados por las características del estudiante, de la familia y de la escuela. Por otra parte, la metodología econométrica aplicada obedece a los modelos multinivel os modelos jerárquicos, los cuales permiten realizar el análisis tanto a nivel individual como por escuela.

También se utilizaron métodos de investigación etnográficos, usando la entrevista como el método de recolección de la información. Con esto se busca conocer la opinión de los distintos agentes involucrados con la educación, en los temas referentes a la calidad educativa y el rendimiento académico.

Debido a todo lo anterior, con esta investigación se busca responder a la siguiente pregunta:

¿Cuáles son los factores que determinan el rendimiento académico de la educación media en Nieva (caracterizada por ser una ciudad con bajo nivel de desarrollo socioeconómico) durante el periodo 2008-2011?. Para resolver esto, se utilizaron los datos de los alumnos de grado once o último año de bachillerato de colegios del sector público del casco urbano de la ciudad de Neiva.

El documento se divide en seis apartados. El primer expone la introducción; en el segundo se discute el planteamiento del problema; en el tercero se examina el referente teórico; el cuarto apartado se centra en la metodología; el quinto recoge los resultados obtenidos en el análisis empírico; y en el sexto y último, se presentan las principales conclusiones del trabajo. 


\section{Planteamiento del Problema}

Al revisar los resultados las Pruebas Saber $11^{\circ}$ desde el 2000 hasta el 2011, se observa que los puntajes promedio a nivel nacional, departamental y municipal tienen comportamientos similares. El promedio de la prueba de lenguaje a nivel municipal se ubicó por encima del promedio nacional y departamental para todo el periodo estudiado, a excepción del año 2009 donde el promedio nacional superó al promedio municipal y departamental. La década empezó con promedios relativamente bajos (46 puntos); fue aumentando paulatinamente hasta llegar a su punto más alto en toda la década, donde Neiva alcanzó un promedio de 52,44 puntos, 0,89 puntos más que el promedio departamental y 0,25 más que el promedio nacional. A partir de allí los promedios empezaron a disminuir, sin embargo, en 2010 volvió a tener un sobre salto, pero en el 2011 el departamento presentó uno de los promedios más bajos para todo el periodo estudiado (ver Figura 1). El punto relevante en esta situación, es el bajo promedio nacional en las pruebas, muy distante del promedio de las principales ciudades, comportamiento que el resta relevancia a que el promedio nacional siempre haya estado por debajo del promedio municipal y departamental, debido a que Colombia es un país con grandes desigualdades económicas y sociales, en donde este bajo promedio nacional es jalonado por las regiones más pobres del país; aunque también se debe recalcar que la diferencia entre los tres niveles es poco significativa.

Figura 1: Pruebas Saber $11^{\circ}$ : promedio lenguaje

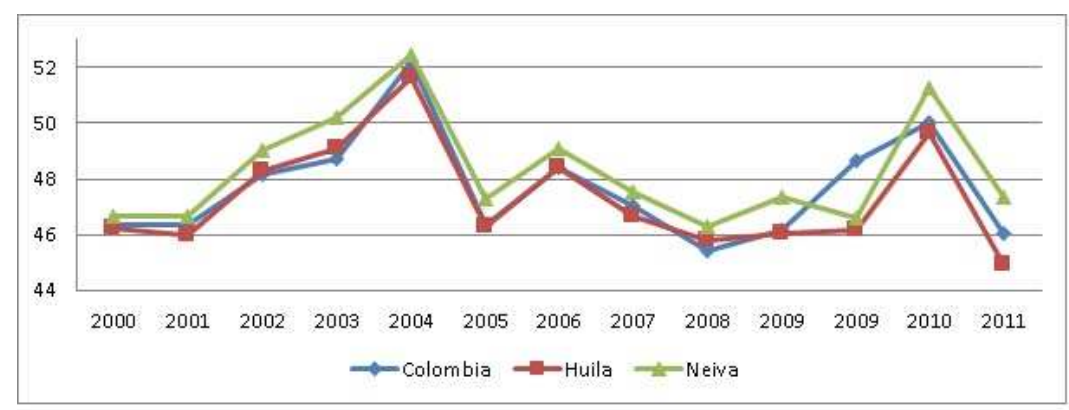

Fuente: Elaboración propia con base en los resultados históricos del ICFES. Excel.

Pero los resultados empeoran cuando se observa los puntajes de la prueba de matemáticas; el Figura 2 registra el comportamiento de los promedios nacional, departamental y municipal en la prueba de matemáticas para el mismo periodo anterior. Del 2000 al 2009 el promedio para cualquier nivel no superó los 47 puntos, siendo el 2004 el año en que peores resultados se registraron (41,04 puntos a nivel nacional, 40,77 a nivel departamental y 41,13 a nivel municipal). De nuevo el puntaje promedio municipal siempre estuvo por encima del promedio nacional y departamental a excepción del año 2009 donde el puntaje promedio nacional fue ligeramente superior. La diferencia promedio para todo el periodo de estudio entre Neiva y Colombia apenas llega a 0,69 puntos, mientras que entre Neiva y Huila asciende a 0,98 puntos.

El bajo puntaje promedio presentado en cada nivel es preocupante; en este caso, el promedio de matemáticas a nivel municipal registrado en las Pruebas Saber $11^{\circ}$ llego a 44,92 puntos, 0,98 puntos por encima del promedio departa- 
Figura 2: Pruebas Saber $11^{\circ}$ : promedio matemáticas

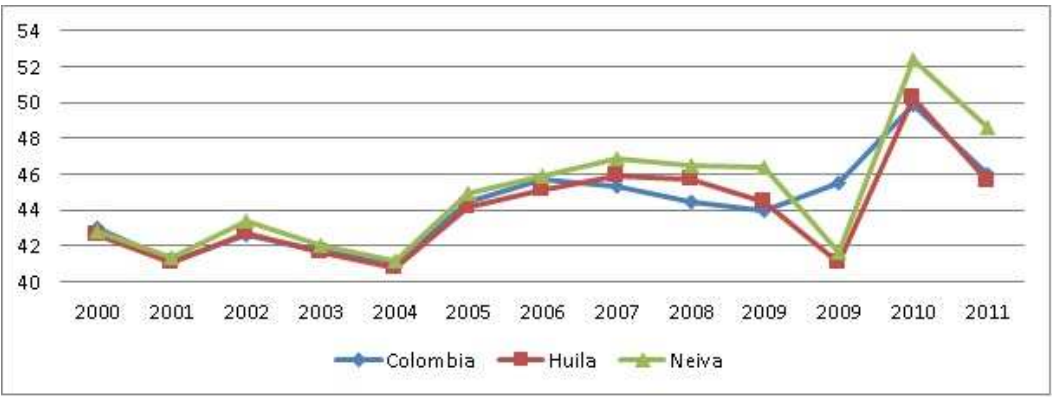

Fuente: Elaboración propia con base en los resultados históricos del ICFES. Excel.

mental y 0,69 por arriba del promedio nacional. Las pruebas en matemáticas presentan en promedio 3,4 puntos menos que las pruebas en lenguaje, ambas por debajo de los 50 puntos.

Según cifras de la Secretaria de Educación Municipal, en cuanto al rendimiento académico de los colegios para el periodo 2001-2010, sólo $7 \%$ de las instituciones estuvieron en la categoría muy superior, $8 \%$ en la superior, $16 \%$ en la categoría alto, $35 \%$ en el nivel medio y $34 \%$ en el nivel bajo e inferior. Estos resultados muestran una posición favorable con respecto a los promedios departamentales, aunque no deja de preocupar, la cantidad de instituciones categorizadas en los niveles bajo e inferior y la alta dispersión entre categorías.

El municipio de Neiva es una región que se caracteriza por presentar altos niveles de desigualdad y pobreza (en el 2011 el 24,9\% de la población es pobre y el 3,9\% se encuentra en situación de indigencia); es una de las ciudades que registra las mayores tasas de inflación y desempleo del país $(5,41 \%$ y $11,8 \%$, respectivamente durante 2011 , en ambos casos por encima del promedio nacional); la actividad económica está representada especialmente por el sector primario, en donde los sectores de explotación de minas y canteras y la agricultura, tienen la mayor participación en el PIB. La actividad empresarial se basa fundamentalmente en las organizaciones unipersonales de tipo familiar (el 97\% de las empresas son microempresas con menos de 6 trabajadores). Todos estos elementos permiten clasificar a Neiva, como una región de bajo desarrollo y crecimiento económico.

Resulta interesante preguntarnos si existe alguna relación entre el panorama socioeconómico de Neiva y el rendimiento académico de los estudiantes. De acuerdo con otros estudios (Mina et al. 2004, Caro et al. 2000, Fuchs \& Wössmann 2005), existe evidencia empírica acerca de la relación positiva entre el crecimiento y desarrollo económico y social, con el desempeño académico y/o resultados escolares de una determinada región.

Autoras como Inés Aguerrondo, caracterizan la calidad de la educación como un concepto complejo totalizante y multidimensional, como concepto social e históricamente determinado, como concepto que se constituye en imagen-objeto de transformación educativa, concepto que se constituye en un patrón de control, sirve de norte y patrón de comparación para ajustar decisiones y reajustar procesos (Jiménez \& Velásquez 2008). Al ser la calidad educativa un concepto de amplias dimensiones, ella es determinada por un conjunto amplio de variables; por lo tanto, los resultados educativos varían dependiendo del contexto en que se desarrollen. Regiones con altas tasas de crecimiento 
económico, diversidad productiva, índices favorables de competitividad, bajos indicadores de desigualdad y pobreza, mayores oportunidades de empleo, mayor acceso a salud y servicios básicos, adecuado entorno académico, entre otros aspectos, tendrá una población con mejores condiciones de vida, niños bien alimentados, sin conflictos familiares, y en condiciones favorables para la enseñanza educativa y el desempeño académico.

De acuerdo con lo anterior, y teniendo en cuenta las características socioeconómicas de Neiva, con el presente artículo se busca determinar el impacto sobre el rendimiento académicos (medido a través de los puntajes en pruebas estandarizadas) de las características individuales y personales o características de Nivel 1, y de las características de la escuela o características de Nivel 2.

Por lo tanto, conociendo la realidad económica y social del Municipio de Neiva, y el bajo rendimiento escolar, se busca responder preguntas como: ¿a qué se deben estas diferencias en el rendimiento escolar?, ¿Cuáles son los factores individuales o de la familia que determinan un mayor o menor puntaje?, ¿La escuela tiene un peso importante sobre el rendimiento académico?. Además, se busca alcanzar el siguiente objetivo: "Determinar los factores que influyen en el rendimiento académico de la educación media para el sector de educación pública del municipio de Neiva durante el periodo 2008-2011".

\section{Referente Teórico}

\subsection{Referentes Documentales}

El aprendizaje estudiantil depende de muchos factores que en algunos casos no presentan aparentes relaciones, pero que en la práctica pueden influir; estos elementos van desde la educación del padre y los valores de la sociedad, hasta la infraestructura escolar y el calendario agrícola (Vegas \& Petrow 2008). El último elemento parecería curioso, pero si se piensa en una región rural, donde económicamente depende de la agricultura, en épocas de cosechas toda la población, incluido niños y maestros, se dedican a la recolección de los productos, bien sea por escases de mano de obra, por necesidades económicas o porque el costo de oportunidad de hacer cualquier otra actividad es mayor (remuneraciones altas).

Desde la publicación del Informe Coleman, en 1966, en los estudios sobre calidad educativa se ha encontrado consistentemente que las condiciones socioeconómicas de los estudiantes y sus antecedentes familiares causan los mayores efectos sobre el rendimiento académico. Estudios posteriores a Coleman (en Inglaterra el informe Plowden y en Francia el estudio longitudinal 1962-1972, que condujo el Institut National Etudes Démographiques-NED) demostraron de forma clara el peso del origen social sobre los destinos escolares, llegando a la misma conclusión de Coleman.

Según Vegas \& Petrow (2008), la edad de entrada a la primaria puede afectar la trayectoria y los logros educativos de un niño en el sistema educativo; pero esta correlación tiene diferentes implicaciones en diferentes países. En Estados Unidos, los padres tienden a retardar la entrada de sus hijos a la escuela primaria con el fin de aumentar sus oportunidades de éxito en el colegio; en países en desarrollo por su parte, la entrada retardada al colegio se debe a factores económicos y sociales. 
Fertig \& Schmidt (2002), utilizando los datos individuales de PISA 2000, analizan cómo se relacionan los resultados en la prueba de lectura con la información individual y los antecedentes familiares y con las características de la escuela; encuentran que la educación materna tiene un mayor efecto en el aprendizaje general del niño, pero que la educación paterna es más importante cuando han llegado al nivel de educación terciaria. Caro et al. (2000), en un estudio realizado para la ciudad de Bogotá, determina los factores asociados al logro académico de los alumnos de $3^{\circ}$ y $5^{\circ}$ de primaria. Al igual que Fuchs \& Wössmann (2005) una fuerte correlación entre los antecedentes socioeconómicos y el resultado escolar; además encuentra que el ausentismo y el trabajo infantil tienen efectos negativos en el desempeño académico de los alumnos.

Gómez et al. (2010), usando los resultados en las áreas de lenguaje y matemáticas de las Pruebas Saber $11^{\circ}$ para el segundo semestre del año 2009, identifican los determinantes del rendimiento académico en Colombia utilizando un modelo Logit Ordenado Generalizado. Los autores comprobaron que las variables socioeconómicas juegan un papel primordial en la calidad de la educación; tener condiciones económicas favorables afecta en gran medida la probabilidad de obtener un puntaje medio y alto tanto en matemáticas como en lenguaje, esto puede explicarse debido a que un entorno socioeconómico favorable le permite al estudiante contar con las condiciones esenciales para dedicarse a sus estudios. En este sentido, variables como tener computador en casa, nivel de ingreso y nivel de escolaridad de los progenitores se relacionan positiva y significativamente con el resultado escolar. Padres con mayor nivel educativo pueden apoyar favorablemente el proceso de enseñanza y aprendizaje de sus hijos; este mayor nivel educativo por lo general se asocia con un mayor nivel de ingreso necesario para adquirir la infraestructura suficiente como computadores, libros, etc. Los autores también concluyen que por lo general los hombres se desempeñan mejor en el área de matemáticas, mientras que las mujeres lo hacen mejor en lenguaje.

Según Sanders \& Rivers (1996), las características y el comportamiento del personal docente, en especial de los maestros, influyen de manera considerable en el aprendizaje estudiantil. Un maestro ineficaz reduce potencialmente el desempeño de un estudiante durante años, pero varios maestros bajo las mismas características multiplican el efecto. Algo claro es que determinar las características y comportamientos de los maestros eficaces o ineficaces es un tema complejo, debido especialmente a la escasez de información, en especial en los países en desarrollo, debido a esto son pocas las experiencias que avalan el impacto de variables específicas del maestro sobre el resultado escolar.

"Calidad de la Educación y Rendimiento Académico en Bogotá", estudio realizado por Gaviria \& Barrientos (2001), analizan los efectos del entorno familiar y las características del plantel sobre el rendimiento escolar en Bogotá, con base en los resultados de las Pruebas Saber $11^{\circ}$ para el año 1999. Tres conclusiones principales se desprenden del trabajo, i) la educación de los padres tiene un efecto sustancial sobre el rendimiento académico, ii) el efecto de la educación de los padres se transmite principalmente a través de la calidad de los planteles educativos y iii) la incidencia de los planteles sobre el rendimiento supera la incidencia conjunta de las características familiares y es mayor que la observada en otros países. Según los autores, estas tres premisas sugieren que, la existencia de restricciones en el acceso a la buena educación constituye un factor muy importante de inmovilidad social en Colombia.

Las tecnologías de información y comunicaciones (TIC) tienen gran poten- 
cial para mejorar los procesos de aprendizaje. Según Hepp et al. (2004), la inversión en TIC en la educación se considera como una forma primaria de preparar a las personas para las nuevas necesidades del mercado laboral y permitir que regiones rezagadas desarrollen las competencias necesarias para igualar las oportunidades de acceso al empleo. Fuchs \& Wössmann (2005)), hallaron que el uso de computadores en el colegio no tiene efecto alguno sobre el puntaje del PISA; por el contrario, el uso de computador en casa, en especial con acceso a internet, correo electrónico y software educativo, garantiza un mayor rendimiento académico.

Piñeros \& Pinzón (1999), encuentran que "las escuelas tienen un efecto pequeño pero considerable sobre el logro académico, teniendo entre $15 \%$ y $18 \%$ de la varianza entre alumnos en escuelas privadas y $12 \%$ y $16 \%$ en escuelas públicas". Otro de los hallazgos del estudio son las grandes diferencias de desempeño en las pruebas que hay entre y dentro de las escuelas especialmente del sector privado; esto puede deberse a las diferencias en dotación física de los establecimientos educativos. La infraestructura académica y los materiales didácticos muestran una correlación positiva con el logro escolar; por tanto, laboratorios de ciencias, bibliotecas, instalaciones deportivas, tiene un efecto estadística y empíricamente significativo y positivo sobre el rendimiento académico de los alumnos; es decir, un suministro adecuado de insumos repercute positivamente en el logro escolar. Los autores también sostienen que los estudiantes matriculados en escuelas de jornada completa muestran un rendimiento significativamente mayor que los estudiantes matriculados en escuelas con jordanas de medio día.

López (2010), a partir de las Pruebas Saber $11^{\circ}$ desde 1980 a 2009, estima el porcentaje de la variabilidad del logro académico atribuible al plantel educativo dentro de un análisis multinivel discriminado por sector educativo. El "efecto colegio" ha sido siempre mayor para el promedio total de la prueba que para el área de matemáticas. Otro punto importante es el cambio de forma y fondo realizado a las pruebas de Estado después de la última década; estos cambios de metodología incidieron en los resultados de las pruebas, pues los puntajes promedios bajan drásticamente en especial en el sector público lo que produce que el coeficiente del factor colegio baje considerablemente en ambos sectores, siendo constantes hasta el final de la década; entonces a partir del 2000 se modificó la importancia del colegio en el rendimiento académico de los estudiantes colombianos.

A diferencia de lo anterior, en nuestro estudio se propone realizar el análisis de los rendimientos en la educación a partir de un modelo multinivel, en una región como Neiva, ciudad intermedia de Colombia, caracterizada por presentar bajo nivel de desarrollo económico y social; mientras que la mayoría de estudios se aplican en las ciudades principales (generalmente las de mayor nivel de desarrollo económico y social), o a nivel agregado de países olvidando que al interior de los mismo existen grandes diferencias.

\subsection{Marco Teórico}

El aporte de Hanushek (1986) es una referencia obligada para el tratamiento económico de la educación ya que da las pautas y menciona los problemas a los que se puede enfrentar el investigador, "la economía puede analizar la educación con un enfoque de insumo-producto, es decir, un marco en el cual la 
calidad de la educación es determinada por una serie de factores" (Hanushek 1986).

Una función de producción en educación se refiere al análisis estadístico que permite observar los resultados de los estudiantes ${ }^{1}$, en función de un conjunto de características que pueden ser propias como la edad, el sexo, características familias y de la escuela. De lo expuesto se desprende que su uso en el campo educativo ha sido potencial para el análisis y definición de políticas.

El Figura 3, representa la función de producción en educación desarrollada por Hanushek en 1978. En este tipo de investigaciones, el nivel de desagregación generalmente se presenta a nivel del estudiante (tomando promedios escolares, test en las asignaturas necesarias, resultado de pruebas estandarizadas, etc.) o captando promedios por zonas.

Figura 3: Diagrama de insumos y resultados en educación

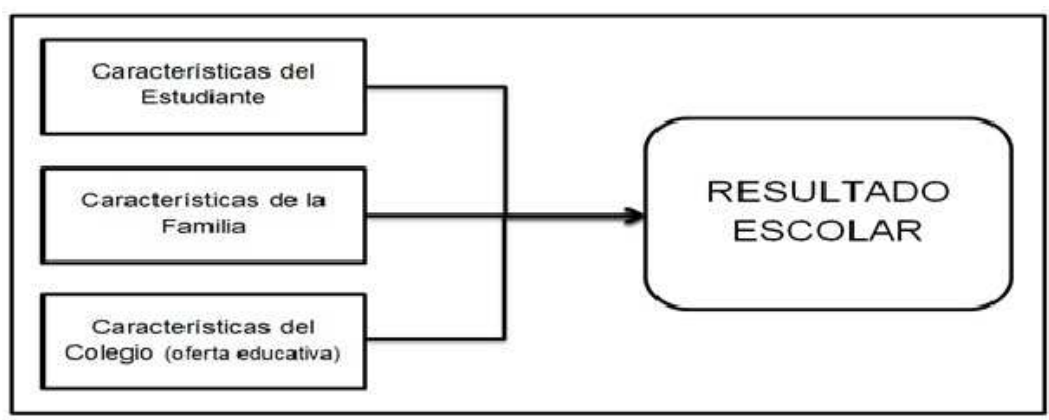

Fuente: Tomado de Hanushek (1986).

El modelo conceptual generalmente aceptado para establecer la función de producción en educación tiene la siguiente especificación:

$$
R_{i t}=f\left(F_{i}^{(t)}, P_{i}^{(t)}, O_{i}^{(t)}, I_{i}\right)
$$

Donde, $i$ representa el $i$-ésimo estudiante y $t$ el periodo de tiempo,

$R_{i t}=$ resultado escolar en el tiempo $\mathrm{t}$

$F_{i}^{(t)}=$ vector de características familiares en el tiempo $\mathrm{t}$

$P_{i}^{(t)}=$ vector de compañeros de aula en el tiempo $t$

$O_{i}^{(t)}=$ vector de insumos escolares en el tiempo $t$

$I_{i}=$ vector de habilidades innatas

Esta forma funcional relaciona los resultados escolares a nivel del estudiante con grupos de variables o insumos con los cuales se establece una relación teórica directa (familia, compañeros, insumos escolares y habilidades innatas).

\footnotetext{
${ }^{1}$ Estos resultados se derivan por lo general de las pruebas estandarizadas aplicadas a los estudiantes.
} 


\section{Diseño Metodológico}

El desarrollo metodológico del presente estudio demando la implementación de dos tipos de análisis. Por un lado, se utilizaron métodos de investigación etnográficos, que consiste en descripciones detalladas de situaciones, eventos, personas, interacciones y comportamientos que son observables. Incorpora lo que los participantes dicen, sus experiencias, actitudes, creencias, pensamientos y reflexiones tal como son expresadas por ellos mismos y no como uno los describe (González et al. 2004). Se utilizó la entrevista, con el objetivo de conocer las opiniones de los diferentes agentes ${ }^{2}$ que tienen que ver con la educación, sobre temas relacionados con la calidad educativa, el rendimiento académico y las Pruebas Saber $11^{\circ}$. Se aplicaron en total 43 entrevistas, distribuidas así: directivos (2), rectores (4), profesores (11), estudiantes (16), padres de Familia (10).

Posteriormente se desarrollaron métodos de investigación cuantitativa. Para la aplicación de este método se consultó dos fuentes de información: i) al ICFES quien suministró la información de orden personal y socioeconómico de los individuos, junto con su respectivo resultado en las Pruebas Saber $11^{\circ}$ para cada uno de los años objeto de análisis; ii) a la Secretaria de Educación de Neiva, donde distribuyeron la información referente a la planta docente de cada una de las instituciones públicas de educación media de la ciudad.

Una vez compilada la base de datos, el paso a seguir fue caracterizar la población objeto de estudio a través de la estadística descriptiva. Posteriormente, se estimó un modelo multinivel de dos niveles explicativos (nivel estudiante y nivel escuela) del rendimiento en lenguaje y matemáticas usando el puntaje en la prueba Saber $11^{\circ}$, con el objeto de determinar cuáles de las variables escolares e individuales afectan el rendimiento académico de los estudiantes. Para el desarrollo estadístico, se consideró un número total de estudiantes de 11.195, distribuidos así 2.758 en 2008, 3.050 en 2009, 2.804 en 2010 y 2.583 en 2011; los estudiantes estuvieron matriculados en las 23 instituciones de educación pública que se analizaron durante todo el periodo de estudios.

Las Pruebas Saber $11^{\circ}$ presentan un puntaje que va desde 1 hasta 100, y se clasifican en cinco niveles: inferior, bajo, medio, alto, superior y muy superior. La formulación y aplicación de dichas pruebas está a cargo del Instituto Colombiano para el fomento de la Educación Superior, ICFES, la cual es una entidad especializada que evalúa la educación en todos sus niveles, y en particular apoya al Ministerio de Educación Nacional en la realización de los exámenes de Estado y en la realización de investigaciones sobre los factores que inciden en la calidad educativa. Las Pruebas Saber $11^{\circ}$, antes llamada Pruebas de Estado, se reglamentaron bajo el Decreto 2343 de 1980, la presentación del examen es obligatorio por parte de todos los estudiantes que cursan el último año escolar de la educación media, con el propósito fundamental de comprobar los niveles mínimos de aptitudes y conocimientos de los estudiantes. A lo largo del tiempo, las Pruebas Saber $11^{\circ}$ han cambiado de metodología, siempre intentando medir de la manera más eficientes el rendimiento académico. No obstante, desde el 2008 la prueba no sufre modificaciones considerables, razón por la cual, el periodo de estudios 2008-2011, no presenta problemas de comparabilidad.

\footnotetext{
${ }^{2}$ Se entrevistaron a directivos, rectores, profesores, estudiantes y padres de familia de la ciudad de Neiva. Las entrevistas estaban compuestas por un núcleo común y uno específico para cada agente.
} 


\section{Resultados}

\subsection{Análisis de las Entrevistas}

Los resultados de las entrevistas realizadas, permiten argumentar que es preocupante la concepción de calidad educativa que tienen los agentes entrevistados. Un poco más de la mitad de los rectores, profesores y padres de familia no consideran en sus análisis "la formación integral" dentro de la educación de calidad. Todas las personas entrevistadas insisten en que una educación de calidad tiene inmersos principios y valores, pero descuidan elementos que se desarrollan cuando existe una educación de calidad, como las capacidades, destrezas y actitudes necesarias para equiparar a los jóvenes durante su vida adulta.

Se resalta que el $25 \%$ de los docentes y rectores, así como el $10 \%$ de los estudiantes consideren que no existe una relación entre calidad educativa y rendimiento académico, cuando dicha relación es clara, pues en cierta medida la calidad educativa determina el rendimiento.

Los directivos, un número significativos de estudiantes y padres de familia consideran que la evaluación interna que realiza la institución educativa es coherente con la evaluación externa que realiza el ICFES. El problema con esta afirmación es que según ellos dicha relación se explica por su parecido en el tipo de preguntas que aplican. Se supone que las Pruebas Saber $11^{\circ}$ están diseñadas para medir ciertas competencias y saberes de los estudiantes; su diseño $\mathrm{y}$ aplicación tienen una regularidad de alto nivel que la hace diferente a una simple preguntas con respuesta de selección múltiple. Esto lo que demuestra es que existe un desconocimiento total por el tipo de preguntas que realiza ICFES, es probable que directivos y padres de familia nunca hayan leído una pregunta de la prueba Saber $11^{\circ}$ y que los estudiantes recién las conozcan el día que presentan la prueba, por tanto, ante este desconocimiento es difícil hacer una comparación clara y coherente entre los métodos internos y externos de evaluación.

Es interesante señalar que los directivos, rectores, docentes y estudiantes dicen conocer la estructura e intencionalidad de las Pruebas Saber $11^{\circ}$, pero realmente no tienen claro cuál es la intención de la prueba ni mucho menos su estructura. Respeto a la intencionalidad se limitan a comentar que es una prueba que evalúa el conocimiento de los estudiantes, sirve para ingresar a la educación superior y miden ciertas competencias que en muy pocos casos mencionan. En cuanto a la estructura no se hicieron referencias claras.

De acuerdo con los determinantes de la calidad educativa, todos los agentes le dan prioridad especialmente a los factores de la escuela (infraestructura y profesores) y de la familia, aspectos importantes en la generación de una educación de calidad. Hay ciertos factores a los que no les dan mayor importancia, como el aporte del estudiante, los factores sociales, las condiciones socioeconómicas entre otras.

Respecto al papel que juega el gobierno departamental y municipal en el mejoramiento del rendimiento académico, consideran que el mismo no solo se limita a acompañar el proyecto educativo en general, sino también a intervenir en el mejoramiento de los ambientes escolares (tanto de planta física como de profesores) necesarios para un mejor desempeño académico tanto de estudiantes como de instituciones educativas. Sin duda el papel del Estado es importante, pero a nuestro parecer descuida elementos fundamentales que 
también contribuyen en la calidad educativa y el rendimiento académico de los estudiantes tales como el mejoramiento de las condiciones socioeconómico de los estudiantes.

La mayoría de los profesores coinciden en afirmar que los métodos de evaluación los diseñan pensando en que van a cumplir con los lineamientos de las Pruebas Saber $11^{\circ}$. Resulta interesante preguntarse si es pertinente educar a los estudiantes pensando en que deben responder eficientemente a una prueba, o si el tipo de preguntas que los mismos docentes desarrollan cumplen con las características de las preguntas implementadas por el ICFES.

Tres cuartas partes de los estudiantes están de acuerdo con la manera como los evalúan en la institución educativa, pues según ellos, en la evaluación se tratan los temas aprendidos en clases. Otro número considerable de estudiantes manifiesta que la evaluación interna es tipo ICFES, esto les permite repasar para la prueba Saber $11^{\circ}$. Tanto los estudiantes como los profesores, olvidan ciertos aspectos que deben contener los procesos de evaluación, necesarios para garantizar una adecuada formación académica.

\subsection{Análisis Estadístico Descriptivo}

El análisis estadístico descriptivo permite caracterizar la población objeto de estudio, en este caso, los estudiantes del grado once de las instituciones de educación pública de la ciudad de Neiva.

El Cuadro 1 muestra el porcentaje de estudiantes analizados distribuidos a lo largo del periodo evaluado (última columna). Se puede observar una distribución con proporciones similares y cierta tendencia a la baja a medida que transcurren los años. Esta reducción en el número de estudiantes, se puede explicar por la disminución en las tasas de matrícula que se registraron en los últimos años en la ciudad. Del cuadro también se observan los puntajes promedios por área de conocimiento evaluada para cada uno de los años; los puntajes de lenguaje y matemáticas tendieron a aumentar, siendo más significativo el aumento en matemáticas, donde el puntaje promedio para el año 2011 llego a 48,55 puntos, 2,81 puntos por encima del puntaje promedio registrado en el año 2008.

Cuadro 1: Puntaje promedio por año y área, 2008-2011

\begin{tabular}{c|cc|cc|c}
\hline AÑO & \multicolumn{2}{|l|}{ Puntaje Matemáticas } & \multicolumn{2}{|l|}{ Puntaje Lenguaje } & \% Estudiantes \\
\hline 2008 & 45,74 & - & 46,15 & - & $24,64 \%$ \\
2009 & 45,18 & $-0,56$ & 46,94 & 0,80 & $27,24 \%$ \\
2010 & 46,43 & 0,69 & 46,21 & 0,07 & $25,05 \%$ \\
2011 & 48,55 & 2,81 & 47,21 & 1,06 & $23,07 \%$ \\
\hline
\end{tabular}

Fuente: Elaboración propia con base en los resultados históricos del ICFES. Software SPSS 15.

En el 2008 el 55\% del total de estudiantes eran mujeres, mientras que para el 2011 la cifra aumentó a 58\%. Los hombres $(47,64)$ tienden a obtener un puntaje promedio más alto en matemáticas que las mujeres $(45,48)$ tal y como se muestra en otros estudios; mientras que en lenguaje tanto hombres $(46,63)$ como mujeres $(46,62)$ tienden a obtener resultados similares.

La edad promedio de los estudiantes durante el periodo 2008-2011 es de 16,76 años. El $97 \%$ de los estudiantes tiene 19 años o menos, y el $82 \%$ tiene 
menos de 17 años. A medida que la edad de los estudiantes aumenta, el puntaje promedio para las dos áreas evaluadas disminuye; pero esta diferencia es más pronunciada en matemáticas que en lenguaje.

El $47 \%$ de los padres de familia de los estudiantes de grado once de la ciudad de Neiva, tienen un nivel educativo de secundaria, el 32,9\% alcanzaron primaria. Solo el 9,15\% de los progenitores tiene algún tipo de educación superior. El impacto de la educación del padre de familia sobre el rendimiento de los estudiantes es mayor en matemáticas que en lenguaje. Los estudiantes cuyo padre tienen nivel de educación primaria $(44,97)$, obtienen en promedio 4,39 puntos menos en matemáticas que los estudiantes con padre de nivel de educación superior; mientras que los estudiantes con padre de nivel de educación superior $(48,79)$, obtiene en promedio 3,16 puntos más en lenguaje que aquellos estudiantes cuyo papá solamente alcanzó el nivel de educación de primaria.

El $54,4 \%$ de los estudiantes de grado once de la ciudad de Neiva, tienen madres con nivel de educación de secundaria, el 28,8\% tienen madres que solamente alcanzaron el nivel de educación de primaria. La proporción de padres con nivel de educación superior $(9,15 \%)$ es mayor a la proporción de madres con el mismo nivel de educación $(8,1 \%)$. La evaluación de la madre tiene un mayor impacto en el rendimiento de matemáticas del estudiante. Los estudiantes con madres de nivel de educación superior $(49,55)$, obtienen en promedio 4,92 puntos por encima del puntaje promedio de estudiantes con madres de nivel de educación de primaria, y 5,53 puntos más que aquellos estudiantes con madres que no presentan ningún nivel de educación en el área de matemáticas. Los alumnos con madres que no registran ningún nivel de educación $(44,74)$, obtienen en promedio 2 puntos menos en lenguaje que aquellos alumnos con madres de nivel de educación secundario, y 4 puntos menos que las madres con nivel de educación superior.

El 95,23\% del total de estudiantes pertenecen a estratos socioeconómicos ${ }^{3}$ 1 y 2, mientras que sólo el $0,68 \%$ pertenecen a los estratos 4,5 y 6 . Como era de esperarse, las características en las regiones poco desarrolladas y con problemas económicos y sociales, la mayoría de su población se clasifican entre los estratos socioeconómicos más bajos. Los estudiantes de estrato socioeconómico 6 obtienen en promedio 48,33 puntos en lenguaje, 2,5 puntos por encima de los de estrato 1 , y 1,32 puntos más que los estudiantes de estrato 2 . En cuanto a los resultados de matemáticas, los estudiantes de estrato $3(48,03)$ obtienen en promedio 3 puntos más que los estudiantes de estratos 1, y 0,94 puntos más que los estudiantes de estrato 2 .

Un poco más del $80 \%$ de los alumnos convive en hogares que cuentan con menos de 2 salarios mínimos legales vigentes. El 51,78\% de los estudiantes vive en hogares que cuentan con ingresos entre uno y menos de dos salarios mínimos. Sólo el 14,22\% de los hogares analizados, reciben como ingresos entre dos y menos de tres salarios mínimos; a su vez el 5,44\% de los hogares reciben ingresos mayores a 3 salarios mínimos. Del Figura 4 se observa que, a mayores ingresos económicos del hogar, mayores rendimientos académicos se registran.

Los estudiantes con computador en el hogar, obtiene en promedio 47,4

\footnotetext{
${ }^{3}$ Los estratos socioeconómicos son una clasificación de los hogares que realiza el Estado colombiano con base en las características socioeconómicas de los hogares. La clasificación va de 1 a 6 , siendo 1 el menor.
} 
Figura 4: Diferencias de puntaje por ingresos del hogar, 2008-2011

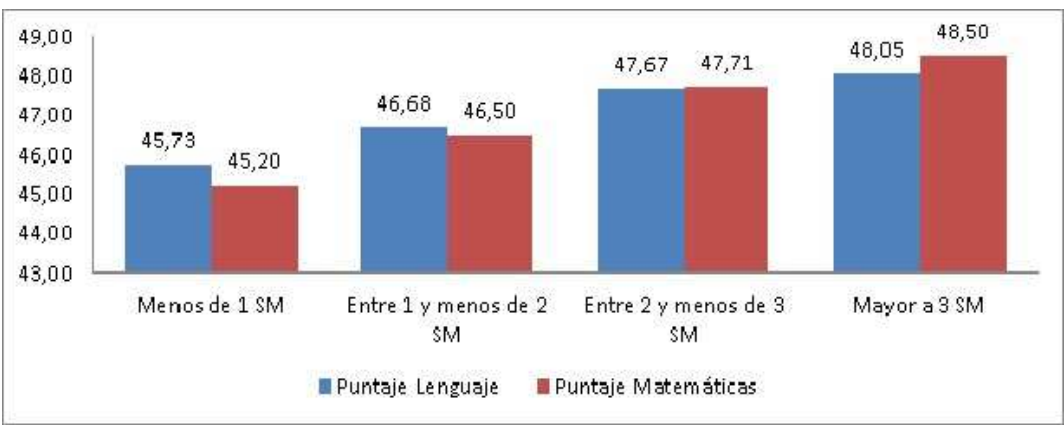

Fuente: Elaboración propia con base en los resultados históricos del ICFES. Excel.

puntos en lenguaje, 1,49 puntos por encima de aquellos estudiantes que no tienen computador. El efecto sobre el puntaje de matemática es mayor, los alumnos con computador en el hogar obtuvieron 47,72 puntos, 2,51 puntos más que los alumnos que no tienen computador. Tener internet en casa implica obtener en promedio 47,33 puntos en matemáticas, 1,6 puntos por encima de los estudiantes sin acceso a internet. En el área de lenguaje, dicha diferencia alcanza los 1,42 puntos.

Entre el año 2008 a 2011, en promedio trabajo el 7,2\% del total de estudiantes por año. Del total de estudiantes que trabajan, el 45,78\% son mujeres, y el porcentaje restantes son hombres. El 88,51\% de los estudiantes mayores de 22 años trabajan, y obtienen en promedio menos puntaje que aquellos estudiantes que no trabaja. Este efecto es mayor en matemáticas, debido a que los estudiantes que trabajan $(44,34)$ obtienen en promedio 2,23 puntos menos que aquellos que no trabajan. En lenguaje, los estudiantes que no trabajan obtienen 1,96 puntos más que aquellos estudiantes que sí lo hacen $(44,81)$.

Cerca del $46 \%$ de los estudiantes pertenecen a la jornada mañana, un $30,92 \%$ pertenecen a la jornada de la tarde, el $20,10 \%$ a la jornada completa, y el $2,99 \%$ a la jornada nocturna o sabatina. Los estudiantes de jornada completa obtienen en promedio 47,07 puntos en lenguaje, 0,49 puntos por encima de los estudiantes de la jornada mañana y 4,23 puntos por encima de los estudiantes de la jornada nocturna o sabatina. En matemáticas, los estudiantes de la jornada tarde obtienen un puntaje promedio de $(47,21), 7,67$ puntos más que los estudiantes de la jornada nocturna y sabatina.

El $67,59 \%$ de los estudiantes, asiste a clases en instituciones de educación técnica, y el 26,86\% lo hace en instituciones de carácter académico, el porcentaje restante lo hace en instituciones normalistas. Los establecimientos educativos técnicos le dan mayor énfasis a las matemáticas razón por la cual alcanzan un puntaje promedio de 47 puntos, 1,97 puntos por encima de las instituciones de carácter académico y 1,53 puntos más que los establecimientos normalistas. Por otro parte, el puntaje promedio en lenguaje es más alto en las instituciones normalistas $(47,40)$ que en las demás.

Cerca del $67 \%$ de los estudiantes no había presentado la prueba previamente, mientras que el $27,26 \%$ presentó la prueba una vez, y un poco más del $5 \%$ lo hizo en más de dos ocasiones. Un estudiante que nunca rindió la prueba Saber $11^{\circ}$ previo a la presentación oficial tenida en cuenta en este estudio, obtiene en promedio 45,29 puntos en matemáticas, 2,78 puntos menos que quienes la presentaron una vez, 5,83 puntos menos que aquellos estudiantes 
que rindieron la prueba dos veces, y 8,51 puntos por debajo de los estudiantes que presentaron la prueba tres veces o más. El puntaje promedio de lenguaje de los estudiantes que nunca presentaron la prueba, alcanza los 45,9 puntos, 1,95 puntos menos que aquellos estudiantes que presentaron la prueba una vez, y 4,41 puntos por debajo de los alumnos que rindieron la prueba tres veces o más.

Al observar el puntaje promedio de matemáticas distribuidos por grupos de estudiantes, los resultados indican que grupos de alumnos de matemáticas de 20 a 25 personas, obtienen un puntaje promedio de 48,13 puntos, 4,2 puntos por encima de grupos con menos de 15 estudiantes, y 2,14 puntos más que los grupos con más de 30 alumnos. En cuanto al puntaje promedio de lenguaje, se encontró que los grupos de alumnos de lenguaje con más de 30 estudiantes obtuvieron un puntaje promedio de 47,16 puntos, un punto más que los grupos con menos de 15 estudiantes, y 0,15 puntos por encima de grupos de alumnos entre 20 y 25 individuos.

\subsection{Análisis Multinivel}

En los inicios de la investigación sobre eficacia escolar y rendimiento educativo se utilizó el modelo de regresión múltiple con datos de estudiantes sin agregar, mediante un procedimiento de mínimos cuadrados Coleman et al. (1966) y Jencks et al. 1972). Esta estrategia olvida el contexto en el que la conducta se desarrolla. Esto implica que la matriz de varianza - covarianza de los resultados no refleja la homogeneidad intragrupo, es decir, olvida la correlación intragrupo.

Los modelos multinivel resuelven este y otros problemas que se presentan al momento de aplicar el método de mínimos cuadrados. Con estos modelos se resuelve el dilema entre agregación disgregación al combinar simultáneamente varios niveles (Cabré et al. 2008). Del mismo modo, permite establecer relaciones entre variables de distintos niveles. Además, con estos modelos es posible detectar diferentes fuentes de variación a través de los distintos niveles y a través de las distintas variables predictoras que se van incluyendo progresivamente en forma de efectos fijos o efectos aleatorios (Suárez et al. 2009).

De acuerdo con Goldstein (1995), los sistemas de escolarización presentan un claro ejemplo de una estructura jerárquica o multinivel, con las estudiantes agrupados o anidados dentro de las escuelas, que a su vez pueden ser agrupados dentro de un territorio, que a su vez pueden ser agrupados dentro de un país.

Para este análisis se definieron dos niveles: el Nivel 1 o micro definido por los estudiantes y el Nivel 2 o macro definido por los colegios, se tiene:

Nivel 1:

$$
Y_{i j}=\alpha_{0 j}+\beta_{1 j} X_{i j}+\varepsilon_{i j} \quad \text { Variables Individuales }
$$

Nivel 2:

$$
\alpha_{0 j}=\gamma_{00}+\gamma_{01} W_{j}+U_{0 j} \beta_{1 j}=\gamma_{10}+\gamma_{11} W_{j}+U_{1 j} \quad \text { Variables Grupales }
$$


Remplazando (2) en (1), y reordenando, se obtiene la formulación convencional de un modelo multinivel, en donde al principio se colocan los efectos fijos, y al final los efectos aleatorios (entre paréntesis):

$$
Y_{i j}=\gamma_{00}+\gamma_{01} W_{j}+\gamma_{10} X_{i j}+\gamma_{11} X_{i j} W_{j}+\left(U_{1 j} X_{i j}+U_{0 j}+\varepsilon_{i j}\right)
$$

En donde, $Y_{i j}$ representa el puntaje en la prueba Saber $11^{\circ}$ del estudiante $i$ que pertenece al colegio $j$ para las áreas de matemáticas y lenguaje; $X_{i j}$ representa el conjunto de variables de Nivel 1 que caracterizan al estudiante $i$ que pertenece al colegio $j ; W_{j}$ son el conjunto de variables de Nivel 2 que caracterizan la institución educativa $j ; \gamma_{00}$ es el rendimiento medio en la población de escuelas; $U_{0 j}$ rendimiento diferencial de la escuela $j ; \gamma_{01}$ es el incremento o disminución en el rendimiento esperado de la escuela por cada unidad que aumente $W ; \gamma_{10}$ es el incremento en el rendimiento del estudiante por el aumento de $X$ en la población de escuelas; $U_{1 j}$ es el incremento diferencia en el rendimiento de estudiante por el aumento en $X$ en la escuela $j ; \gamma_{11}$ es el incremento o disminución en el rendimiento por cada cambio en $X$ y $W$; $\varepsilon_{i j}$ variación residual del estudiante $i$, es decir lo que se aparta del rendimiento esperado según su $X$, donde $\varepsilon_{i j} N\left(0, \sigma_{\varepsilon}^{2}\right)$, se asume varianza homogénea para todas las escuelas.

\section{Análisis de Datos}

El modelado multinivel suele componerse de una serie de pasos comunes recogidos entre distintos autores (Heck \& Thomas 2015). So \& Kim (2009); Murillo (2007); Raudenbush \& Bryk (1992)); en vista de esto, a continuación, se estiman una serie de modelo partiendo del más simple al más complejo. Estos modelos se estimaron utilizando el software IBM SPSS Statistics 19.

El modelo multinivel más simple se obtiene substrayendo del modelo (3) el conjunto de variables independientes $X$ y $W$. De esta forma se obtiene un modelo sin variables independiente que recibe el nombre de modelo incondicional o nulo.

Las estimaciones del modelo nulo, que incluye el efecto del factor colegio, arrojaron unos valores para el -2LL1 de -9011,041 para lenguaje y -3124,649 para matemáticas, este valor obedece al modelo que incluye la constante y el factor de efectos aleatorios. El efecto del colegio puede evaluarse comparando el modelo que incluye ese efecto con el modelo que no lo incluye. El valor del estadístico $-2 \mathrm{LL}^{4}$ del modelo que únicamente incluye la variable dependiente y la constante asciende a $-8591,82$ para lenguaje y $-2498,38$ para matemáticas $^{5}$. La diferencia entre ambos valores es de 419,22 en el caso de lenguaje y 626,26 en el caso de matemáticas, estos valores se distribuyen según chicuadrado con 1 grado de libertad (el que corresponde al parámetro asociado al factor colegio, que es el único parámetro en el que difieren ambos modelos). La probabilidad de encontrar valores chi-cuadrado iguales o mayores a 455,61 y 673,91 es menor a 0,0005; por lo tanto, se puede rechazar la hipótesis nula de que el efecto del factor colegio es nulo.

\footnotetext{
${ }^{4}$ Estadísticos de ajuste global indican en qué medida el modelo propuesto es capaz de representar la variabilidad observada en los datos.

${ }^{5}$ Para obtener estos valores basta con ajustar el modelo que únicamente incluye la variable dependiente (puntleng y punmat) y el término constante.
} 
El Cuadro 2, muestra el valor estimado de la constante, que es el único parámetro de efectos fijos que presentan estos dos modelos (el que utiliza el logpuntleng y el logpuntmat como variable dependiente). Esta constante no es otra cosa que la estimación de la media poblacional de las 23 instituciones educativas en el rendimiento de lenguaje $(\hat{\mu}=3,8166)$ y matemáticas $(\hat{\mu}=$ $3,7917)^{6}$. Dicha estimación aparece acompañada de su error típico, los grados de libertad su valor tipificado ( $t$ que se obtiene dividiendo la estimación entre su error típico) y el nivel crítico obtenido al contrastar la hipótesis de que el parámetro vale cero. En este caso esto nos indica que el puntaje promedio tanto de lenguaje como de matemáticas es mayor a cero.

Cuadro 2: Estimación de los parámetros de efectos fijos modelo AEA

\begin{tabular}{lcccccc}
\hline Modelo & Parámetro & Estimación & Error Típico & $g l$ & $t$ & Sig. \\
\hline $\begin{array}{l}\text { Puntaje } \\
\text { Lenguaje }\end{array}$ & Intersección & 3,816676 & 0,008876 & 21,872 & 430,013 & 0 \\
\hline $\begin{array}{l}\text { Puntaje } \\
\text { Matemáticas }\end{array}$ & Intersección & 3,791713 & 0,013024 & 21,875 & 291,135 & 0 \\
\hline $\begin{array}{l}\text { Fuente: Elaboración propia con base en los resultados históricos del ICFES. Software } \\
\text { SPSS 15. }\end{array}$ & & & & & & \\
\end{tabular}

El Cuadro 3 ofrece las estimaciones de los parámetros de covarianza, es decir, las estimaciones de los parámetros asociados a los efectos aleatorios del modelo. La varianza del factor colegio indica cuanto varía la variable dependiente entre los colegios de toda la población, para el puntaje de lenguaje la varianza del factor colegio es 0,001725 y para matemáticas es de 0,003753. La varianza de los residuos indica cuanto varía la variable dependiente dentro de cada colegio, para el puntaje de lenguaje la varianza de los residuos es 0,02599 y para matemáticas es de 0,04395. Los resultados indican que hay una mayor varianza entre y dentro de los colegios en el puntaje de matemáticas que en el puntaje de lenguaje. La variabilidad entre las instituciones educativas en el rendimiento en lenguaje representa el $\frac{0,001725}{0,001725+0,02599}=0,0622$, o $6,22 \%$ de la variabilidad total, mientras que en matemáticas representa el $\frac{0,003753}{0,003753+0,04395}=0,07867$ o $7,867 \%$ de la variabilidad total. Este coeficiente recibe el nombre de Coeficiente de Correlación Intraclase ICC, que de acuerdo con Lee (2001) presenta el grado variabilidad existente entre los distintos colegios en comparación con la variabilidad existente entre los estudiantes del mismo colegio; el análisis del ICC es importante en el uso de métodos de varios niveles, pues responde a la pregunta ¿qué proporción de la varianza total de la variable dependiente se encuentra de forma sistemática entre los grupos?.

Las estimaciones del Cuadro 3 permite contrastar la significancia de cada estimación. Se quiere contrastar la hipótesis $H_{0}: \sigma_{\beta}^{2}=0$, de que la varianza poblacional del factor colegio vale cero. Para esto se utiliza el estadístico $Z$ de Wald. Dado que la significancia es menor a 0,05, se puede rechazar la hipótesis nula y afirmar que la varianza del factor escuela es distinta de cero. De esta forma se concluye que el rendimiento académico no es el mismo en todas las instituciones académicas.

\footnotetext{
${ }^{6}$ Este valor expresa la media en logaritmo; en valores naturales la media del puntaje de logaritmo es de 46,06 y la media del puntaje de matemáticas es de 45,35.
} 
Cuadro 3: Estimación de los parámetros de covarianza AEA

\begin{tabular}{|c|c|c|c|c|c|c|}
\hline Modelo & \multicolumn{2}{|c|}{ Parámetro } & | Estimación & | Error Típico & Wald Z & Sig. \\
\hline \multirow{2}{*}{$\begin{array}{l}\text { Puntaje } \\
\text { Lenguaje }\end{array}$} & Residuos & \multirow[b]{2}{*}{ Varianza } & 0,025990 & 0,000348 & 74,739 & 0,000 \\
\hline & \begin{tabular}{|c|} 
Institución \\
Educativa \\
\end{tabular} & & 0,001725 & 0,000547 & 3,151 & 0,002 \\
\hline \multirow{2}{*}{$\begin{array}{l}\text { Puntaje } \\
\text { Matemáticas }\end{array}$} & Residuos & \multirow[b]{2}{*}{ Varianza } & 0,043951 & 0,000588 & 74,739 & 0,000 \\
\hline & $\begin{array}{l}\text { Institución } \\
\text { Educativa }\end{array}$ & & 0,003753 & 0,001179 & 3,184 & 0,001 \\
\hline
\end{tabular}

Fuente: Elaboración propia con base en los resultados históricos del ICFES. Software SPSS 15.

Cuadro 4: Estimación de los parámetros de efectos fijos modelo RMR

\begin{tabular}{l|c|c|c|r|r|r}
\hline Modelo & Parámetro & Estimación & Error Típico & \multicolumn{1}{c}{$g l$} & \multicolumn{1}{c}{$t$} & Sig. \\
\hline \multirow{3}{*}{ Puntaje } & Intersección & 3,709356 & 0,013528 & 98,121 & 274,196 & 0,000 \\
Lenguaje & Jornada Completa & 0,126696 & 0,01589 & 276,242 & 7,973 & 0,000 \\
& Jornada Mañana & 0,10481 & 0,010035 & 10741,995 & 10,444 & 0,000 \\
& Jornada Tarde & 0,101565 & 0,010134 & 10734,155 & 10,022 & 0,000 \\
\hline \multirow{3}{*}{ Puntaje } & Intersección & 3,618309 & 0,022562 & 81,916 & 160,370 & 0,000 \\
Matemáticas & Jornada Completa & 0,243559 & 0,021891 & 443,490 & 11,126 & 0,000 \\
& Jornada Mañana & 0,182783 & 0,013005 & 11051,216 & 14,055 & 0,000 \\
& Jornada Tarde & 0,197169 & 0,013133 & 11035,358 & 15,013 & 0,000 \\
& Ratio Estu.Prof. & $-0,001254$ & 0,00052 & 1067,251 & $-2,415$ & 0,016 \\
\hline
\end{tabular}

Fuente: Elaboración propia con base en los resultados históricos del ICFES.

Software SPSS 15.

Ya se comentó que existen diferencias entre las medias de los colegios que ofrecen grado once en la ciudad de Neiva; ahora se estimó el modelo: medias como resultados (RMR) con el que se busca determinar si esta variación se debe a la incorporación de variables de Nivel 2. Para esto se agregaron inicialmente las variables ratio estudiante/profesor, Jornada Académica de la Institución Educativa, tipo de bachiller de la Institución Educativa. De las primeras estimaciones resultaron ser no significativas el tipo de bachiller de la institución educativa en ambos modelos, y la ratio estudiante/profesor en el modelo de lenguaje.

El Cuadro 4 muestra el resultado de dichas estimaciones. La primera parte del cuadro describe el modelo RMR utilizando como variable dependiente el logaritmo del rendimiento en lenguaje. La intercepción arrojó un resultado de 3,709 , mientras que las variables explicativas de Nivel 2 resultaron ser todas significativas tanto estadística como empíricamente. Por ejemplo, el valor del coeficiente de la variable jornada completa indica que las instituciones educativas que ofrecen jornada completa obtienen en promedio un $12,6 \%$ más puntaje en lenguaje que las instituciones que ofrecen jornada nocturna y sabatina.

El Cuadro 4, también describe el modelo RMR utilizando como variable dependiente el logaritmo del rendimiento en matemáticas. Las instituciones educativas que ofrecen jornada completa obtienen en promedio un $24,35 \%$ más puntaje en matemáticas que aquellas instituciones que ofrecen jornada nocturna o sabatina. Del mismo modo la ratio estudiante/profesor de matemáticas se relaciona negativamente con el rendimiento en matemáticas, un estudiante adicional por clase de matemáticas disminuye el puntaje promedio por institución en $0,125 \%$.

El Cuadro 5 muestra las estimaciones de los parámetros de covarianza. En 
el modelo de lenguaje, la estimación de la varianza de los residuos $(0,02573)$ es similar a la obtenida en el modelo nulo (0,02599); por lo tanto, no parece que la variabilidad de Nivel 1 se vea afectada por la presencia de la variable tipo de jornada académica. Del mismo modo la variabilidad entre los colegios tampoco ha sufrido grandes variaciones paso de 0,001725 en el modelo AEA a 0,001698 en el modelo RMR, por lo tanto, la variabilidad del Nivel 2 se ha visto afectada, pero en menor proporción por la presencia del tipo de jornada de cada institución educativa. El nivel crítico asociado al estadístico de Wald $($ Sig. $=0,002)$ indica que después de controlar por el conjunto de variables de Nivel 2, se rechaza la hipótesis nula de que la variación en el puntaje promedio entre colegios es igual a cero.

Cuadro 5: Estimación de los parámetros de covarianza RMR

\begin{tabular}{|c|c|c|c|c|c|c|}
\hline Modelo & \multicolumn{2}{|c|}{ Parámetro } & | Estimación & Error Típico & Wald Z & Sig. \\
\hline \multirow{2}{*}{$\begin{array}{l}\text { Puntaje } \\
\text { Lenguaje }\end{array}$} & Residuos & \multirow[b]{2}{*}{ Varianza } & 0,025734 & 0,000344 & 74,732 & 0,000 \\
\hline & $\begin{array}{c}\text { Institución } \\
\text { Educativa }\end{array}$ & & 0,001698 & 0,000541 & 3,137 & 0,002 \\
\hline \multirow{2}{*}{$\begin{array}{l}\text { Puntaje } \\
\text { Matemáticas }\end{array}$} & Residuos & \multirow[b]{2}{*}{ Varianza } & 0,043026 & 0,000576 & 74,722 & 0,000 \\
\hline & $\begin{array}{l}\text { Institución } \\
\text { Educativa }\end{array}$ & & 0,004817 & 0,001572 & 3,064 & 0,002 \\
\hline
\end{tabular}

Fuente: Elaboración propia con base en los resultados históricos del ICFES. Software SPSS 15.

Para el caso de matemáticas, la estimación de la varianza de los residuos $(0,043026)$ varía relativamente poco en relación con la obtenida en el modelo nulo $(0,043951)$, por lo tanto, no parece que la variabilidad de Nivel 1 se vea afectada por la presencia de las variables que indican la jornada académica y la ratio estudiante/profesor. Por otro lado, la estimación de la variabilidad entre los colegios pasó de 0,003753 en el modelo nulo a 0,004817 en el modelo RMR, esta variación permite concluir que la variabilidad de Nivel 2 se vio ligeramente afectada por la inclusión de las variables de Nivel 2. El nivel crítico asociado al estadístico de Wald (Sig.=0.001), indica que después de controlar por las variables de nivel dos, los colegios difieran en el rendimiento promedio de matemáticas. De nuevo se observa que el efecto colegio en mayor en matemáticas que en lenguaje.

Debido a los resultados anteriores, se debe tener cuidado con el análisis del cambio en las varianzas de los dos modelos al incluir las variables de nivel dos; el poco efecto de las variables de nivel dos sobre la variabilidad de Nivel 1 puede deberse a que al incluir las variables de Nivel 2, al estar agrupadas, disminuyen el número de escuelas utilizadas en el análisis; los grados de libertad de la distribución de los componentes de la varianza dan una idea de esta situación.

Si se comparan los estadísticos -2LL, que permiten ver la significancia estadística de las varianzas, se concluye que la varianza entre los colegios es distinta de cero, pues tanto el -2LL de lenguaje $(-9101,266)$ como de matemáticas $(-3325,728)$ es menor al $-2 \mathrm{LL}$ del modelo nulo, por lo que se concluye que el ajuste global de los dos modelos mejoró con la incorporación de las variables de Nivel 2.

El CCI del modelo RMR para el rendimiento en lenguaje asciende a 6,24\%, mientras que para el modelo de matemáticas llega a 10,06\%. Esta variación 
Cuadro 6: Estimación de los parámetros de efecto fijos modelo RCA

\begin{tabular}{l|c|c|l|r|r|c}
\hline Modelo & Parámetro & Estimación & Error Típico & $g l$ & \multicolumn{1}{c}{$t$} & Sig. \\
\hline \multirow{3}{*}{ Modelo de } & Intersección & 3,80434 & 0,007873 & 18,166 & 483,210 & 0,000 \\
Lenguaje & Presen_Prueba & 0,03234 & 0,005207 & 11,087 & 6,211 & 0,000 \\
& Estutrabaja & $-0,027395$ & 0,00824 & 17,971 & $-3,325$ & 0,004 \\
& InternetHog & 0,012964 & 0,003671 & 19,349 & 3,532 & 0,002 \\
\hline \multirow{3}{*}{ Modelo de } & Intersección & 3,747666 & 0,012056 & 21,306 & 310,855 & 0,000 \\
Matemáticas & Presen_Prueba & 0,049986 & 0,007007 & 12,780 & 7,133 & 0,000 \\
& Genero & 0,056901 & 0,012431 & 21,088 & 4,577 & 0,000 \\
& EP_Terciaria & 0,045072 & 0,010188 & 11,050 & 4,424 & 0,001 \\
\hline
\end{tabular}

Fuente: Elaboración propia con base en los resultados históricos del ICFES. Software SPSS 15.

fue mayor, de nuevo, en matemáticas. Comparando las estimaciones de los parámetros de covarianza del modelo nulo y del modelo RMR puede conocerse la proporción de varianza explicada en el Nivel 2. De esta forma, el 28,25\% y el $1,56 \%$ en matemáticas y lenguaje respectivamente, de las diferencias observadas entre los colegios son atribuibles a las variables que indican la jornada académica de las instituciones educativas y a la ratio estudiante/profesor de matemáticas (variables de Nivel 2). Es decir, la inclusión de las variables de Nivel 2 al modelo, en comparación con el modelo nulo que no incluyen variables de ningún nivel, produce que la varianza explicada aumente, más en matemáticas que en lenguaje, lo que significa que evidente las variables de Nivel 2 (características del colegio) afectan el rendimiento académico.

El desarrollo de la metodología propone ahora determinar que parte de la variabilidad intracentro (variabilidad de Nivel 1) puede ser explicada por las variables de Nivel 1 que se agregaron al modelo. Para resolver esto, es necesario obtener una ecuación de regresión para cada colegio y analizar como varían las intercepciones y las pendientes de esas ecuaciones. Al proceder de esta manera, se asume no solo que los colegios pueden diferir en el rendimiento académico promedio, sino también que la relación entre el rendimiento promedio y cada una de las variables de Nivel 1 pueden no ser la misma en todos los colegios. Este tipo de modelos es denominado Modelo de Coeficientes Aleatorios, porque deja que la intercepción y la pendiente varíen aleatoriamente de colegio a colegio.

Después de varias estimaciones individuales y grupales incluyendo cada una de las variables independientes, con el propósito de determinar la significancia, no solo estadístico sino también empírica de los parámetros estimados, se construyó el modelo de lenguaje y matemáticas con las variables de Nivel 1 que resultaron tener significancia estadística y empírica.

El Cuadro 6 ofrece las estimaciones de los parámetros fijos de ambos modelos. El valor de los coeficientes asociados a las variables independientes agregadas son todos estadísticamente significativos. En la primera parte se evidencia que el coeficiente de la variable presento la prueba, indica que los estudiantes que presentaron la prueba previamente obtienen un 3,23\% más de puntaje en lenguaje con respecto a quienes no presentaron la prueba antes. El estadístico $t$ y su nivel crítico (Sig. $=0,000$ ) permiten afirmar que el coeficiente es significativamente distinto de cero. Del mismo modo, los estudiantes que trabajan obtienen en promedio un puntaje menor (un 2,73\% menor) que los estudiantes que no trabajan. 
En la segunda parte del Cuadro 6 se muestran los resultados del modelo del rendimiento en matemáticas. Los hombres obtienen en promedio un 5,7\% más de puntaje en matemáticas que las mujeres. Mientras que los estudiantes con padres de nivel de educación terciaria obtienen en promedio $4,5 \%$ más de puntaje que los estudiantes con padres sin educación formal.

Las estimaciones de los parámetros de covarianza para los dos modelos, muestran que la varianza estimada de los residuos del modelo de lenguaje y de matemáticas es $\sigma_{\varepsilon}^{2}$ es 0,025592 y 0,04210 , respectivamente, en ambos casos inferior a la obtenida en el modelo nulo. Al obtener la proporción de la varianza explicada en el Nivel 1 de lenguaje $(0,0153)$ y matemáticas $(0,04211)$, se puede concluir que al incluir variables de Nivel 1 en el modelo de regresión utilizando una ecuación separada para cada colegio, la variabilidad intracentro se reduce aproximadamente $1,53 \%$ y $4,21 \%$, respectivamente.

La varianza de las medias o intercepciones, o variación entre las medias de las instituciones educativas resultaron ser significativas; esto indica que el rendimiento promedio de las instituciones educativas tanto en lenguaje como en matemáticas no es igual en todos los colegios.

La varianza de las pendientes de ambos modelos resultó ser no significativas, por lo tanto, no se puede concluir que las pendientes de las ecuaciones de regresión son diferentes en todos los colegios. Por otro lado, la covarianza entre las medias y las pendientes de ambos modelos para todas las variables de Nivel 1 consideradas en las estimaciones, resultaron ser no significativas, por lo tanto, se concluye que las pendientes no parecen estar relacionadas con las medias. De la aplicación de este modelo, se concluye que la incorporación de las variables de Nivel 1 afecta realmente poco la variabilidad de Nivel 1, siendo mayor en el modelo que utiliza el rendimiento en matemáticas como variable dependiente. De este modo, pueden existir otras variables características del estudiante que no fueron consideradas en estos modelos y que pueden afectar en mayor proporción el rendimiento académico.

Habiendo encontrado que la medias (las constantes e intercepciones) varían entre los colegios, el paso a seguir es averiguar qué variables o qué interacción de variables de distinto nivel podrían dar cuenta de esta variabilidad. Esto se averigua con la aplicación del último modelo análisis de regresión: medias y pendientes como resultados (RMPR). De esta forma, se estiman los modelos finales de lenguaje y matemáticas, que incluyen variables de Nivel 1 y 2, así como variables que indiquen interacciones entre los dos niveles.

Después de una secuencia de estimaciones, en donde se excluyeron las variables que no presentaban significancia estadística y empírica, se estimó el modelo final para cada una de las áreas evaluadas; las ecuaciones 4 y 5 muestra la estimación de los parámetros del modelo final para lenguaje y matemáticas. La omisión de algunas variables que resultaron ser no significativas, no afectaron la bondad del ajuste, pues el estadísticos -2LL se mantuvo en -9120,13 para el caso de lenguaje, y -3549,053 para matemáticas.

$$
\begin{array}{r}
\operatorname{LogRenLeng}_{i t}=3,70436+0,128157 \widehat{J C}_{J}+0,099461 \widehat{J M}_{J} \\
+0,103209 \widehat{J T}_{J}+0,017429 \widehat{H I}_{I J}-0,013581{\widehat{H I_{I J} J C}}_{J} \\
+\left(\varepsilon_{i j}+U_{0 J}+U_{3 J} \widehat{E T}_{I J}\right)
\end{array}
$$




$$
\begin{array}{r}
\text { LogRenMat }_{i t}=3,585969+0,253675 \widehat{J C}_{J}+0,184158 \widehat{J M}_{J} \\
+0,208183 \widehat{J T}_{J}-0,00144 \widehat{R T}_{J}+0,069672 \widehat{G E}_{I J}-0,025465 \widehat{G E} J J T_{J} \\
+\left(\varepsilon_{i j}+U_{0 J}+U_{3 J} \widehat{R T}_{I J}\right)
\end{array}
$$

Las variables indicativas de la jornada académica (variables de Nivel 2) junto con la variable internet en el hogar, y la interacción con la jornada académica tarde (variable de Nivel 1) hacen parte del modelo final que incluye al rendimiento en lenguaje como variable independiente. Por el lado de matemáticas, las variables indicativas de la jornada académica, así como la ratio estudiante/profesor de matemáticas (variables de Nivel 2) y el género, junto con la interacción entre la jornada tarde y el género del estudiante (variables de Nivel 1), integran el modelo multinivel de matemáticas. Estas dos ecuaciones representan los modelos multinivel finales tanto de lenguaje como de matemáticas.

Al comparar el modelo nulo con el actual, se observa que la incorporación de las variables de Nivel 2 incrementaron las varianza de las medias entre los colegios tanto en el rendimiento de lenguaje $(20,92 \%)$ como de matemáticas $(34,1 \%)$; es decir, las variables de Nivel 2 agregadas, explican el 20,92\% y el $34,1 \%$ de las diferencias en el rendimiento de lenguaje y matemáticas entre los colegios, Es evidente que el factor colegio tiene mayor peso sobre el rendimiento de matemáticas que sobre el rendimiento de leguaje.

El CCI aumento levemente con respecto al registrado en el modelo de medias como resultado; para el modelo de lenguaje el CCI alcanzó un valor de $4,82 \%$ mientras que para el modelo de matemáticas dicho valor ascendió a $10,68 \%$. Esto indica que el $10,68 \%$ de la variabilidad total es atribuible a las diferencias entre las medias y/o rendimiento promedio en matemáticas de las instituciones educativas.

\section{Conclusiones}

El desarrollo de la presente investigación, permite formular las siguientes conclusiones:

Del análisis etnográfico se deriva que los agentes entrevistados presentan un alto grado de desconocimiento y desinformación respecto a la calidad educativa, el rendimiento académico y las Pruebas Saber $11^{\circ}$, pues los agentes que tienen que ver con la educación regional presentan una limitada percepción acerca del concepto de calidad educativa, en general se evidencia un total desconocimiento respecto a la intencionalidad y estructura de la prueba Saber $11^{\circ}$, y un gran confusión en relación al tipo de preguntas aplicadas por el ICFES. Esta conclusión concuerda con los resultados de Jiménez \& Velásquez (2008), quienes concluyen que el principal problema de la evaluación de la calidad de la educación en Colombia es que los autores que hacen parte del proceso educativo no conocen el concepto de calidad educativa, y continuamente lo limitan a un concepto puramente cuantitativo.

Si bien, lo que arrojan los datos del ICFES no se discuten, es bueno evaluar como es recibida esta información en la comunidad. Es necesario introducir la prueba en contextos más amplios, y no considerarla como su único fin. Como lo sostiene Aguerrondo (1993), la prueba Saber $11^{\circ}$ tiene que ir más allá 
de la medición y la clasificación de estudiantes, docentes e instituciones. Se debe hacer alusión al comportamiento del sujeto en un orden social dado, en donde se incluyan la formación, apropiación e internalización de valores. Se debe sacar la prueba de esa concepción esencialmente disciplinaria, en donde predomina una posición epistemológica muy distinta a la interdisciplinariedad, la transversabilidad y la integralidad, elementos determinantes en los procesos educativos más complejos.

Tanto la escuela como las características propias del individuo determinan el rendimiento académico de los estudiantes. El factor escuela tiene mayor peso que el factor estudiante, tanto en matemáticas como en lenguaje, ya que las variables de Nivel 2 agregadas, explican el $20,92 \%$ y el $34,1 \%$ de las diferencias en el rendimiento de lenguaje y matemáticas entre los colegios; esto se presenta porque al analizar los estudiantes de las instituciones de educación pública de la ciudad de Neiva, caracterizados por tener bajos niveles de desarrollo socioeconómico, y por no presentar grandes diferencias entre las instituciones, el factor estudiante (la casa, los padres, etc.) difiere poco entre los alumnos, por lo tanto el factor escuela es el que registra mayor variabilidad. De esta forma, al presentar pocas diferencias en las características económicas y sociales de los estudiantes, el factor escuela es quien determina en mayor proporción las diferencias en el rendimiento académico. Estos resultados concuerdan con lo encontrado por López (2010) quien sostiene que el "efecto colegio" ha sido siempre mayor para el promedio total de la prueba en Colombia durante el periodo 1980-2009. Piñeros \& Pinzón (1999), encuentran que el efecto de la escuela sobre el logro académico se encuentra entre $15 \%$ y $18 \%$ de la varianza entre alumnos en escuelas, notablemente inferior al encontrado en la presente investigación.

La situación socioeconómica del estudiante no afecta el rendimiento académico; esto resulta lógico, pues como se mostró en el planteamiento del problema, las características socioeconómicas de los estudiantes de las instituciones de educación públicas de Neiva, no difieren mucho entre ellos, por tanto, no afecta el rendimiento académico de los estudiantes. Estos resultados difieren de los encontrados por Caro et al. (2000) y Fuchs \& Wössmann (2005)), quienes determinan que existe una fuerte correlación entre los antecedentes socioeconómicos y el resultado escolar.

La educación de los padres sin duda afecta el rendimiento académico de los estudiantes; las diferencias en el nivel educativo de los padres no son tan importante en el rendimiento de lenguaje, pero sí en el rendimiento de matemáticas, en donde un mayor nivel académico del padre o la madre, garantiza un mayor logro escolar. Estos resultados coinciden con los resultados de Gómez et al. (2010), quienes sostienen que padres con mayor nivel educativo pueden apoyar favorablemente el proceso de enseñanza y aprendizaje de sus hijos; este mayor nivel educativo por lo general se asocia con un mayor nivel de ingreso necesario para adquirir la infraestructura suficiente como computadores, libros, etc. Gaviria y Barrientos en 2001 para Bogotá concluyen que la educación de los padres tiene un efecto sustancial sobre el rendimiento académico, y que el efecto de la educación de los padres se transmite principalmente a través de la calidad de los planteles educativos.

Del mismo modo, las clases con menor número de estudiantes favorece el rendimiento académico de matemáticas. En la medida que el profesor puede atender de manera personalizada a los estudiantes, la probabilidad de que el estudiante capte mejor los conceptos es mayor. Los estudiantes de género 
masculino presentan mayores rendimientos en matemáticas que los estudiantes de género femenino. Esto no ocurre con el rendimiento en lenguaje, en donde tanto hombres como mujeres registran el mismo resultado académico. El acceso a las tecnologías en especial al internet, favorece el rendimiento en lenguaje. Este resultado refleja la importancia que ha adquirido en los últimos años la tecnología en el desarrollo del proceso educativo. Fuchs \& Wössmann (2005)), hallaron que el uso de computadores en el colegio no tiene efecto alguno sobre el puntaje del PISA; por el contrario, el uso de computador en casa, en especial con acceso a internet, correo electrónico y software educativo, garantiza un mayor rendimiento académico.

Finalmente, se concluye que la problemática a cerca de la calidad educativa, el rendimiento académico y las Pruebas Saber $11^{\circ}$ no está agotada completamente, y menos cuando se estudia regiones como Neiva, que presentan tantas desigualdades económicas y sociales. Esta es apenas un primero estudio que debe servir de base para futuras investigaciones sobre el tema, que tiendan a contribuir en la solución del problema planteado.

En términos de política pública, los resultados anteriores permiten formular una reforma de política educativa encaminada, por un lado, a fortalecer la formación en adultos, para que los padres de familias tengan mayores niveles de educación y de esta forma puedan orientar los procesos de enseñanza de sus hijos. Pero esta formación debe traspasar los niveles de educación media, hasta llegar a la educación superior, partiendo de carreras técnicas y tecnológicas. Y, por otro lado, se deben fortalecer y ampliar programas como "Computadores para Educar", que consisten en dotar a los estudiantes de computadores para que puedan desarrollar sus actividades académicas. Lo anterior debe complementarse con acceso de internet gratuito para toda la comunidad, como una estrategia para mejorar el rendimiento académico de los estudiantes.

Finalmente, debido a la importancia del colegio en el rendimiento académico, deben fortalecerse, no solo con recursos propios sino de las regalías petroleras, ya que por ley una proporción de los mismos deben dedicarse al sector educación, las instituciones de educación no solo con dotaciones físicas, recursos académicos, infraestructura, acceso a tecnologías, sino también con la vinculación de nuevos maestros que permitan disminuir la ratio estudiante/profesor.

\section{Agradecimientos}

Este artículo es producto del proyecto de investigación "Determinantes del rendimiento académico en Neiva", cofinanciado por la Universidad Surcolombiana y Colciencias.

\section{Bibliografía}

Aguerrondo, I. (1993), 'La calidad de la educación: ejes para su definición y evaluación', Revista Interamericana de Desarrollo Educativo 37(116), 561-578.

Cabré, R. B., Seco, G. V. \& Gras, J. A. (2008), 'Técnicas de análisis aplicadas a datos longitudinales en psicología y ciencias de la salud: período 1985-2005', Papeles del Psicólogo . 
Caro, B. L. et al. (2000), Factores asociados al logro académico de los alumnos de 3 y 5 de primaria de Bogotá, Technical report, FEDESARROLLO.

Coleman, J. S. et al. (1966), 'Equality of educational opportunity'.

Easterly, W. (1993), 'How much do distortions affect growth?', Journal of Monetary Economics 32(2), 187-212.

Fertig, M. \& Schmidt, C. (2002), 'The role of background factors for reading literacy: straight national scores in the PISA 2000 study'.

Fuchs, T. \& Wössmann, L. (2005), What accounts for international differences in student prformance? A re-examination using PISA data, in 'The economics of education and training', Springer, pp. 209-240.

Gaviria, A. \& Barrientos, J. H. (2001), 'Determinantes de la calidad de la educación en Colombia'.

Goldstein, H. (1995), 'Multilevel statistical models, 2003', Edward Arnold, London 3.

González, P., Mizala, A. \& Romaguera, P. (2004), 'Vouchers, inequalities and the chilean experience', Documento de Trabajo, Centro de Economía Aplicada (CEA), Universidad de Chile .

Gómez, S. M. C., Gutiérrez, D. M. G., Hassan, A. R. et al. (2010), 'Determinantes del rendimiento académico en Colombia: Pruebas ICFES Saber 11'.

Hanushek, E. A. (1986), 'The economics of schooling: production and efficiency in public schools', Journal of Economic Literature 24(3), 1141-1177.

Heck, R. H. \& Thomas, S. L. (2015), An Introduction to Multilevel Modeling Techniques: MLM and SEM Approaches Using Mplus, Routledge.

Hepp, P., Hinostroza, J. E., Laval, E. \& Rehbein, L. (2004), Technology in Schools: Education, ICT and the Knowledge Society, World Bank, Distance \& Open Learning and ICT in Education Thematic Group, Human Development Network, Education.

Jiménez, N. E. L. \& Velásquez, A. V. P. (2008), 'Acerca de la problemática de la evaluación de la calidad de la educación en Colombia', Entornos 1(21), 9-20.

Lee, V. E. (2001), 'What are multilevel questions and how might we explore them with quantitative methods?', Estudos em Avaliação Educacional (24), 3146.

Lucas Jr, R. E. (1988), 'On the mechanics of economic development', Journal of Monetary Economics 22(1), 3-42.

López, S. F. M. (2010), 'El efecto colegio en Colombia: tres décadas de estudio', Equidad E Desarrollo (14), 85-101.

Mina, A. et al. (2004), Factores asociados al logro educativo a nivel municipal, Technical report, Universidad de los Andes - CEDE. 
Murillo, F. J. T. (2007), 'Los modelos jerárquicos lineales aplicados a la investigación sobre eficacia escolar', Revista de Investigación Educativa, RIE 17(2), 453-460.

Piñeros, L. J. J. \& Pinzón, A. R. (1999), 'Los insumos escolares en la educación secundaria y su efecto sobre el rendimiento académico de los estudiantes: un estudio en Colombia', Washington, DC: The World Bank/Latin America and the Caribbean Region/Department of Human Development .

Raudenbush, S. W. \& Bryk, A. S. (1992), Hierarchical Linear Models: Applications and Data Analysis Methods, Vol. 1, Sage.

Rebelo, S. (1991), 'Long-run policy analysis and long-run growth', Journal of Political Economy 99(3), 500-521.

Romer, P. M. (1986), 'Increasing returns and long-run growth', Journal of Political Economy 94(5), 1002-1037.

Sanders, W. L. \& Rivers, J. C. (1996), 'Cumulative and residual effects of teachers on future student academic achievement'.

So, H.-J. \& Kim, B. (2009), 'Learning about problem based learning: student teachers integrating technology, pedagogy and content knowledge', Australasian Journal of Educational Technology 25(1), 101-116.

Suárez, E. P., Alonso, R. F. \& Fernández, J. M. (2009), 'Estimación del valor añadido de los centros educativos', Aula Abierta .

Vegas, E. \& Petrow, J. (2008), Incrementar el Aprendizaje Estudiantil eb America Latina: el Desafid para el Siglo XXI, The World Bank. 
